\title{
DEVELOPMENT OF CHATTER-RESISTANT SYSTEM OF CUTTING TOOL
}

\author{
Jüri Olt ${ }^{\mathrm{a}}$, Viacheslav V. Maksarov ${ }^{\mathrm{b}}$ \\ ${ }^{a}$ Institute of Technology, Estonian University of Life Sciences, 56 Kreutzwaldi Str., EE51014, Tartu, Estonia \\ ${ }^{b}$ Department of Mechanical Engineering, National Mineral Resources University, 21 Line, Vasilevsky Island, St. \\ Petersburg, Russia
}

\begin{abstract}
The aim of this paper is to propose a new chatter-resistant system of the cutting tool for machining solid-of-revolution type parts. The chatter-resistant system can be used both for turning external surfaces and boring holes. The simulation of the cutting tool system allows to define the effective technology of the tool design structural properties. The experimental study of the cutting tool system during the work piece machining is aimed at promoting the efficient dynamic damping of oscillations. The oscillation absorption and damping factor is increased due to the large dissipative forces resulting from the resistance of the tool holder material that has texture with different metal deformationconditioned orientations.
\end{abstract}

Keywords: metal cutting; chatter vibration; turning tool; boring tool; absorption coefficient; structure of tool holder material
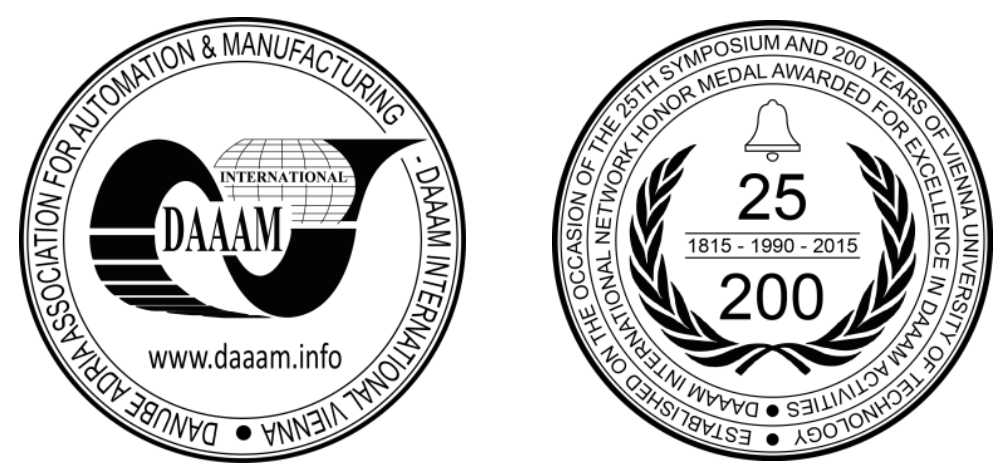

This Publication has to be referred as: Olt, J[ueri] \& Maksarov, V[iacheslav] (2016). Development of ChatterResistant System of Cutting Tool, Proceedings of the 26th DAAAM International Symposium, pp.0223-0228, B. Katalinic (Ed.), Published by DAAAM International, ISBN 978-3-902734-07-5, ISSN 1726-9679, Vienna, Austria DOI:10.2507/26th.daaam.proceedings.031 


\section{Introduction}

Vibrations that occur in the process of machining significantly hinder the use of technological equipment working at an automated cycle, and are the main reason for the premature wear of the tool, machine and equipment accidents. The dynamic durability of the technological system and a decrease in the vibration level (that occurs in the process of cutting) are the key to the stability of chip formation, which is especially important in the case of the automation of the technological process [6], [11]. This way, the task of guaranteeing the stability of the technological system by reducing the level of self-excited oscillations is one of the key issues in edge cutting machining, which becomes particularly topical in the case of the final polishing of products on automated machines, and machines with computer numerical control [3], [5], [9].

The material properties of the workpiece and various other factors can lead to excessive vibrations in the tool shaft, which in turn causes undesirable chattering. By the use of passive damping elements, integrated into the tool holder shaft, the dynamic behavior of the tool can be affected. From the standpoint of dynamic stability we assume that the "tool" is the weakest subsystem of the turning process [11]. The impact of periodical external forces causing an oscillatory process with a frequency equal to the exciting forces or complex periodic processes caused by nonlinear properties of the system, having its own damped and forced oscillations of parametrically excited oscillations and selfoscillation system can violate the stability of the turning system [1].

The intensity of the forced vibrations is particularly great in the resonant region, which must be avoided in metal cutting machines as an operating method for finishing. One aspect of improving the dynamic stability of the subsystem "tool" is the creation of damped tools with increased resistance, with elastic and damping elements that do not change their appearance. It is important for the damped instruments to be characterized by its adaptation to the variable tolerances, load balancing between the cutting edges, as well as to prevent breakage of the cutting edge.

Plenty of fundamental research has been done in the field of study of self-excited oscillations occurring during the cutting of metals in order to understand the chip making processes that occur during turning, and to evaluate the importance of guaranteeing the stability of the technological systems during machining [2], [10], [12], [13]. Owing to the analysis and classification of methods and means of guaranteeing the dynamic stability of technological systems it can be said that, in one or another way, these methods help raising the stability of the technological system, and the means developed on their basis are in a practical manner successfully implemented in machining. However, nowadays there is no universal method that enables to efficiently combat vibrations that appear during turning.

When completing the turning, the weakest link from the point of view of dynamic stability is the "tool" subsystem. The technological system is influenced by external periodic force, causing an oscillation process with a frequency equal to the frequency of perturbing force, or complex periodical processes, conditioned by the non-linear characteristics of the system, as a result of which damping and forced oscillations, parametric induced oscillations and the system self-excited oscillations appear [4]. The intensity of forced oscillations is especially great in the conditions of resonant modes, which are unacceptable for the quality of operating modes during final polishing.

One of the most efficient methods, allowing us to guarantee the stability of the partial technological "tool" subsystem during the final polishing of solid-of-revolution type parts, is the creation of a damping tool system, the effect of which is reached through a combination of the multi-layer principle and the use of anisotropic properties of a rolled metal sheet [7]. The use of such a tool system during tuning operations allows for the absorption of high-frequency oscillations that appear in the process of cutting, through the ordered disorientation of the texture of anisotropic plates of a custom multi-layered tool holder, which allows for the effective dissipation of the energy of oscillating wave transitioning between the plates of the tool holder. This method makes it possible to significantly raise the durability of the cutting edge of the instrument and to broaden technological possibilities of choosing effective cutting modes, guaranteeing the fulfilment of requirements of size and geometric precision as well as the quality of the machined surface [6], [14].

\section{Technical conception of development}

The method of designing the damping tool system based on the multi-layer principle implies the use of anisotropy of the qualities of the plates of the tool holder, created through the use of pressure treatment. The plastic deformation of steel, caused by hot rolling, leads to the change in the direction of the fibers in the macrostructure, structural components and additions along its direction; in addition to the crystallographic texture, a mechanical texture of deformation is created. It is suggested that the cutting tool holder be made from a plate packs that are in contact with each other along the planes, which run parallel to the supporting surface of the tool holder (Figure 1). Plates are cut from a rolled metal sheet with a longitudinal 1, lateral 2 and vertical 3 orientation over a surface relative to the direction of their rolling, and then collected into a pack, based on the disorientation of the angle of the texture between the plates. 


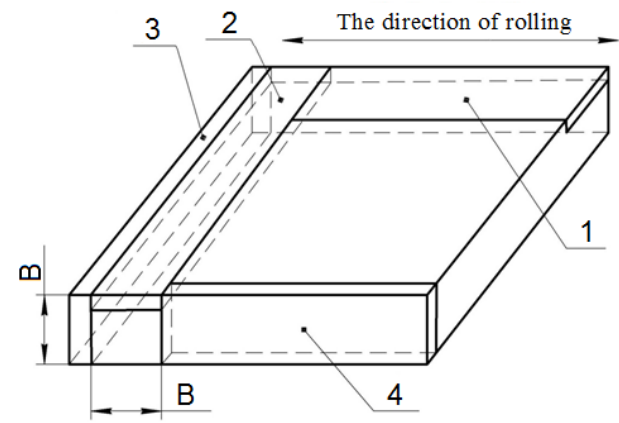

Fig 1. Method of constructing the plates in a damping instrument system.

During oscillations that occur in the process of machining, the characteristics of the behaviour of the tool holder's rod under small deformations can be described using Hooke's law, taking into account the resistance of friction in immobile joints between plates and internal friction in the material of the tool holder. Caused by the texture of the material, the inelastic internal friction effects tied to the movement of dislocations cause irreversible hysteresis losses of energy within the metal of the tool holder during mechanical oscillations [8,9]. In order to achieve the maximal damping effect, the disorientation of the deformation texture in two neighbouring plates must me maximal. In that case, during the transition of the plates, the wave changes direction, as a result of which the oscillation energy shall be dissipated. If the volume of the disorientation of the deformation texture is small, the energy dissipation is minimal. For that reason, in the technical solution presented here the plates of the tool holder must be oriented in a way that the texture would change by $90 \pm 10^{\circ}$ during transitioning from one plate to the other depending on the effect of the main (tangential) component of the cutting force on the cutting tool.

Figure 2 shows the cutting scheme and the directions of cutting forces, according to which the texture of the plates of the cutting tool holder are oriented. All plates have a different deformation texture on their planes and crosssections; they have different physical and mechanical qualities, including damping ones, regarding the activities of the cutting forces acting on the tool holder [5,6]. Under the influence of the cutting force, mainly maximal stretching tensions appear in the upper layers of the tool cutter, and compression tensions in the lower support ones.

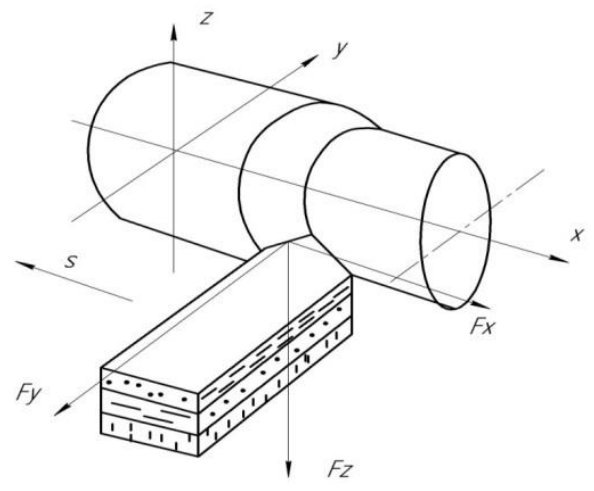

Fig 2. Cutting scheme and the direction of cutting forces, according to which the cutting tool holder plate textures are oriented.

\section{Results of development and discussion}

For stabilising the durability of various zones and increasing the durability and reliability of the entire tool holder, the orientation of deformation texture in the plates of the combination tool holder relative to the cutting forces must meet additional conditions. It is known that metal renders maximum resistance to stretching tensions in the longitudinal direction, and minimal resistance in the vertical direction in view of the direction of rolling. And vice versa, maximum resistance to compressing forces in rolled metal occur in the vertical direction, and minimal resistance in the longitudinal direction. The metal resistance in the lateral direction and resilience to both stretching and compressing tension have intermediate values. The direction of the texture in a rolled metal sheet in a base plate is oriented parallel to the tangential component of the cutting force. In a plate with a fixed cutting tool, the direction of rolling is oriented parallel to the radial component of the cutting force, and the rolling surface is positioned perpendicularly to the tangential component of the cutting force.

Figure 3 shows a scheme of the cutting force directions during the boring of holes and their interconnection to the texture of the plates of the tool holder. In this case, the cutting tool holder is made of plates glued together that are cut from a rolled metal sheet and collected into a pack vertically along the planes relatively to the supporting surface of 
the tool holder, thus guaranteeing that the far left part of the tool holder layer holds back the stretching tensions and the far right part holds back the compression tensions. Thereby, the disorientation of the deformation texture in the two outside plates of the tool holder in relation to the vector of equivalent cutting forces $\mathrm{R}$ between $90 \pm 10^{\circ}\left(\alpha_{\mathrm{p}}<80^{\circ}\right.$ and $\left.\alpha_{p}>100^{\circ}\right)$ is guaranteed. In the middle plate, the texture has a radial direction, guaranteeing the disorientation in comparison to the outer plates of the tool holder.

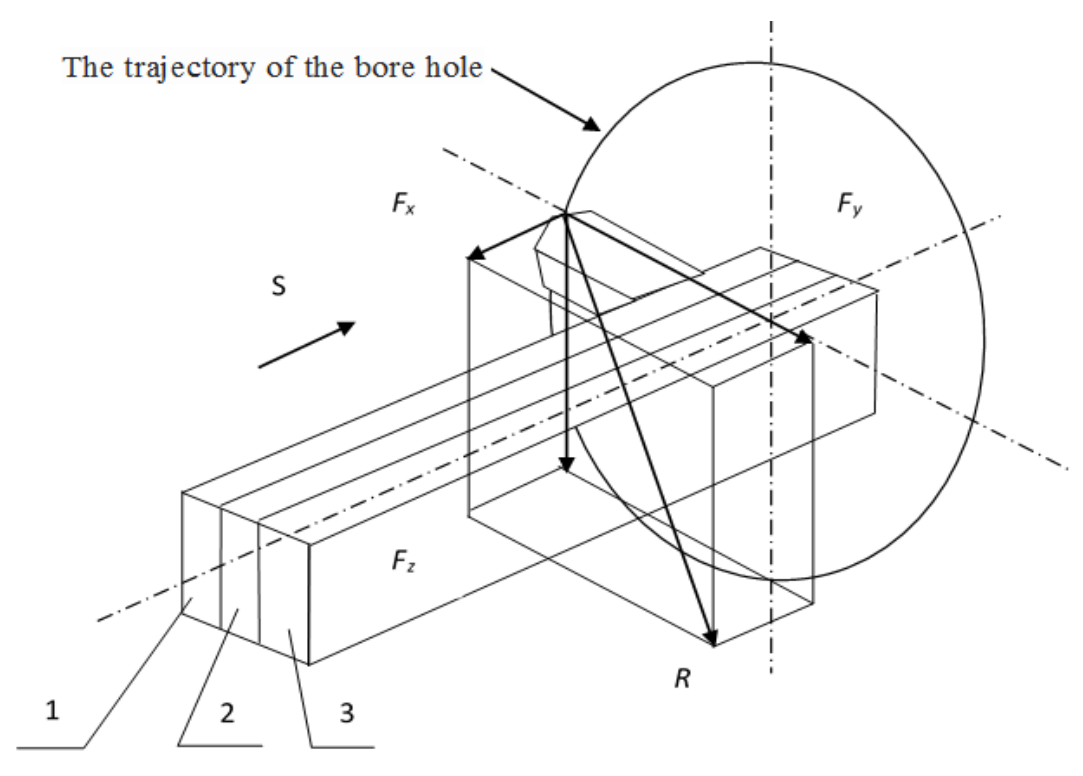

Fig 3. The position of cutting forces during the boring of holes and their interconnection to the texture of the plates of the tool holder.

Plates 1 and 3 are cut out in a way that their surfaces are oriented vertically and the sections perpendicularly to the direction of the rolling and have a longitudinal orientation of the texture in relation to the direction of the rolling. The surface of plate 2 is oriented vertically, across the direction of the rolling, and the surface of its cross-section has a longitudinal orientation. This way, plates 1,3 and plate 2 have a different deformation texture in their surface and crosssection, they have different physical and mechanical properties, including damping ones, in view of the effect of the forces acting on the tool holder (components of cutting forces).

The cut plates are collected into a pack. In order to achieve the greatest damping effect, the disorientation of deformation texture in two neighbouring plates must be maximal. In that case, the oscillation wave changes direction during transition between the plates, as a result of which the oscillation energy is dissipated. In the case of a small disorientation of the deformation texture, the dissipation of energy is insignificant. For that reason the plates of the tool holder are oriented in a way that during the transition from one plate to the other, the texture would change by $90 \pm$ $10^{\circ}$ relative to the effect on the tool holder in view of the main-tangential component of the cutting force in the technological solution that we offer.

Figure 4 shows the actions of the cutting forces in the upper layers of the tool holder, which, in the process of boring, mainly create maximum stretching forces, and compressing tensions in the lower supporting layers.

For that reason, additional conditions requiring the orientation of the deformation texture in the plates of the combination tool holder relatively to the cutting forces must be met, in order to achieve the stabilisation of durability of various zones and increase the durability and reliability of the entire tool holder. It is known that metal renders maximum resistance to stretching tensions in the longitudinal direction, and minimal resistance in the vertical direction in the view of the direction of rolling. On the other hand, rolled metal renders maximum resistance to compressing forces in the vertical direction, and minimal in the longitudinal direction. The metal resistance in the lateral direction and resilience to both stretching and compressing tensions have intermediate values.

For that reason, additional conditions requiring the orientation of the deformation texture in the plates of the combination tool holder relatively to the cutting forces must be met, in order to achieve the stabilization of durability of various zones and increase the durability and reliability of the entire tool holder. It is known that metal renders maximum resistance to stretching tensions in the longitudinal direction, and minimal resistance in the vertical direction in the view of the direction of rolling. On the other hand, rolled metal renders maximum resistance to compressing forces in the vertical direction, and minimal in the longitudinal direction. The metal resistance in the lateral direction and resilience to both stretching and compressing tensions have intermediate values. 


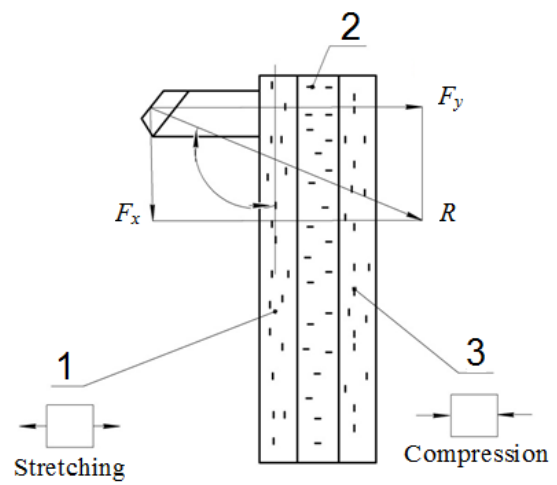

Fig 4. Diagram of cutting forces in the upper layers of the tool holder.

The constructed tool holder consists of three plates, 1-3, with a different deformation texture orientation concerning the effects of cutting forces. Plates 1-3 are glued together vertically relatively to the supporting surface of the tool holder. Plates 1 and 3 have a rolling direction that is oriented perpendicularly to the tangential component of the cutting force $F_{z}$. The direction of rolling in plate 2 is oriented parallel to the radial component of the cutting force $F_{z}$. The rolling surface in plate 2 is oriented perpendicularly to the tangential component of cutting force $F_{z}$.

In this case, the direction of rolling in plates 1 and 3 is oriented parallel to the axial cutting force component $F_{x}$. The lateral direction of rolling in the middle plate 2 is oriented parallel to the tangential cutting force component $F_{z}$. This way, the right angle of texture deformation is maintained between plates 1,3 and 2 . If the force $F_{y}$ is present, this enables to control the stretching forces in the far left part of the layer of the tool holder, and to control the compressing forces in the far right part of the layer of the tool holder. The position of the texture in outer layers of the tool holder along the force $F_{x}$, as well as its constructive position enables to contain the stretching and compressing along the direction of the force $F_{z}$ as well. The texture of the middle part of the layer of the tool cutter must be close to the value of the force $F_{x}$ at nearly $90^{\circ}$.

The energy of tool oscillations appearing in the cutting process is absorbed not only by a viscosity resistant layer of glue, but is also dissipated within the plates that make up the tool holder, as a result of the anisotropy of mechanical and physical features of rolled (textured) materials, including the anisotropy of oscillations and the logarithmic decrements of their own oscillations. During the disorientation of the deformation texture in two neighbouring plates, differentiating by $90 \pm 10^{\circ}\left(\alpha<80^{\circ}\right.$ and $\left.\alpha>100^{\circ}\right)$, the level of energy dissipation decreases, and the technological difficulties of constructing the plates increase (the total amount of operations increases and there is more metal waste).

According to the test results the modified tool compared to an analogue cutter improves the quality of treatment. The vibration data analysis concludes that the modified cutting tool holders have been able to suppress vibrations at high, frequencies above $4000 \mathrm{~Hz}$. Practical issues related to the control selection of the cutting process, are depending on the requirements of dimensional and geometric accuracy of machined surfaces, which can be solved using an instrument equipped with alternate bars. According to the results of experimental studies using a fractional experiment obtained the functional dependence of the actual margin of accuracy of the processed surface.

\section{Conclusion}

The use of the damping tool system during turning operations and the internal boring of highly precise items allows to guarantee a more efficient dynamic damping of oscillations during the process of machining the work pieces than it was possible before, as the absorption coefficient is raised and oscillations are dampened due to large dissipative forces of the resistance of the material of the tool holder that has a texture with different metal deformation-conditioned orientations.

The use of the vibration resistant properties of a damping tool instrument is most acceptable during the final polishing and fine surface turning at high cutting speed modes and a low stiffness of the work piece, with an alternating, continuous or fragmented machining surface, when higher demands regarding geometrical precision of the machining of details and the roughness of the surface are present.

\section{References}

[1] Astakhov, V.P. Tribolology of Metal Cutting. Elsevier, London, (2006) pp. 69 - 219.

[2] Brandon C. Gegg, C. Steve Suh, Albert C. J. Luo. Machine Tool Vibrations and Cutting Dynamics. Springer, New York, (2011) 101-166.

[3] Madissoo, M., Maksarov, V.V., Arak, M., Olt, J. Dynamic modelling of metal turning elements of mechanical processing. DAAAM International Scientific Book, (2012) 213-224. 
[4] Madissoo, M., Rossner, A., Olt, J., Maksarov, V.V. Testing of the oscillations damping capability by external turning with the use of alternate bars in the construction of the turning tool holder. Materials Science (Medžiagotyra), 21(3) (2015) 391-395.

[5] Maksarov, V.V., Olt, J., Leemet, T. Increase in the stability of technological system with control of the process of cutting. International science conference of material science and manufacturing technology, 26-27 June 2008, Prague, Czech Republic, pp. 1-5.

[6] Olt, J., Madissoo, M., Maksarov V.V. Dynamic Stabilization of Technological Systems for Processing Edge Cutting Through the Local Metastability. IEEE International Symposium on Assembly and Manufacturing, 25th27th May 2011, Tampere, Finland.

[7] Olt, J., Maksarov, V.V. Using an anisotropic properties of sheetmetal to develop a design of vibrationless cutting tool. Agronomy Research, 10(1) (2012) 181-186.

[8] Siddhpura, M., Paurobally, R. A review of chatter vibration research in turning. International Journal of Machine Tools and Manufacture, Volume 61, October (2012) 27-47.

[9] Stephenson, David A. , Agapioa, John S. Metal cutting theory and practice. 2nd. ed., Taylor \& Francis Group, LLC, 2006

[10] Tobias S. A. Machine - Tool Vibrations. University of Birmingham, Blackie, 1965.

[11] Tony, L., Schmitz, K., Smith, S. Machining Dynamics. Springer, New York, 2009.

[12] Tony, L., Schmitz, K., Smith, S. Mechanical Vibrations, Springer, New York, 2012.

[13] William C. Elmore, Mark A. Heald. Physics of Waves. Dover edition, New York, 1985.

[14] William D. Callister, David G. Rethwisch. Fundamentals of Materials Science and Engineering: An Integrated Approach, Publisher: Wiley, 2012. 